\title{
Fast 3D Brain Segmentation Using Dual-Front Active Contours with Optional User-Interaction
}

\author{
Hua $\mathrm{Li}^{1,2}$, Anthony Yezzi ${ }^{1}$, and Laurent D. Cohen ${ }^{3}$ \\ ${ }^{1}$ School of ECE, Georgia Institute of Technology, Atlanta, GA, USA \\ 2 Dept. of Elect. \& Info. Eng., Huazhong Univ. of Sci. \& Tech., Wuhan, P.R.China \\ ${ }^{3}$ CEREMADE, UMR 7534 University Paris-Dauphine, 75775 Paris Cedex France \\ hua.li@ece.gatech.edu
}

\begin{abstract}
Important attributes of 3D brain segmentation algorithms include robustness, accuracy, computational efficiency, and facilitation of user interaction, yet few algorithms incorporate all of these traits. Manual segmentation is highly accurate but tedious and laborious. Most automatic techniques, while less demanding on the user, are much less accurate. It would be useful to employ a fast automatic segmentation procedure to do most of the work but still allow an expert user to interactively guide the segmentation to ensure an accurate final result.

We propose a novel 3D brain cortex segmentation procedure utilizing dualfront active contours, which minimize image-based energies in a manner that yields more global minimizers compared to standard active contours. The resulting scheme is not only more robust but much faster and allows the user to guide the final segmentation through simple mouse clicks which add extra seed points. Due to the global nature of the evolution model, single mouse clicks yield corrections to the segmentation that extend far beyond their initial locations, thus minimizing the user effort. Results on 15 simulated and 20 real 3D brain images demonstrate the robustness, accuracy, and speed of our scheme compared with other methods.
\end{abstract}

\section{Introduction}

Three dimensional image segmentation is an important problem in medical image analysis. Determining the location of the cortical surface of the human brain from MRI imagery is often the first step in brain visualization and analysis. Due to the geometric complexity of the brain cortex, manual slice by slice segmentation is quite difficult and time consuming. Thus, many automatic segmentation methods have been proposed which eliminate or nearly eliminate user interaction.

The active contour model has been widely applied in medical imaging, which was introduced in [1] as the "snakes" and is an energy minimization method. Malladi and Sethian [2] showed the initial contributions on 3D segmentation of medical images, after that, numerous contributions [3-8] have been made on the segmentation of complex brain cortical surfaces.

These methods used the active contour model as the final step for the cortex segmentation, applied geometric and anatomical constraints and/or utilized significant pre-processing of the data to obtain desirable final segmentations. Furthermore, curve evolution process in these methods are very time-consuming. 
Some other methods were also proposed for brain segmentation, such as fuzzy set methods [9,10], Bayesian Methods [11], Markov random field methods [1214], expectation-maximization (EM) algorithm [15] and so on. These methods were aimed at segmenting the brain tissues automatically, and limiting the user interaction to choosing the parameters of the automatic process, setting initial surfaces for surface evolution, or restricting regions to be processed. It is usually impossible or very difficult and unintuitive for experts to guide the segmentation process with their professional knowledge for improving the accuracy of the final result. In our opinion, methods that allow simple and intuitive user interaction (while minimizing the need for such interaction as much as possible) are more useful than totally automatic methods given the importance of high accuracy and detail in cortical segmentation.

In this paper, we propose a novel 3D brain cortex segmentation scheme based on dual-front active contours which are faster and yield more global image-based energy minimizers compared to other active contours models. This scheme also adapts easily to user interaction, making it very convenient for experts to guide the segmentation process by adding useful seed points with simple mouse clicks. This scheme is very fast and the total computation time is less than 20 seconds. Experimental results on 15 simulated and 20 real 3D brain images demonstrate the robustness of the result, the high reconstruction accuracy, and the low computational cost compared with other methods.

\section{Cortex Segmentation by Dual-Front Active Contours}

\subsection{D Brain Cortex Segmentation with Flexible User-Interaction}

The basic idea of dual-front active contour model is proposed by the authors in [16] for detecting the object boundaries. Using this model, the segmentation objective is achieved by iteratively dilating the initial curve to form a narrow region and then finding the new closest potential weighted minimal partition curve inside by dual front evolution.

Due to the complex and convoluted nature of the cortex and the partial volume effects of MRI imaging, the segmentation of cortex must be considered in three dimensions. Here we propose the 3D brain cortex segmentation scheme based on the dual-front active contours. This scheme is simple, fast, and accurate with flexible user-interaction. In Fig 1, we show an overall diagram of this scheme. And we will give the details about choosing the active region and the potentials in the following subsection.

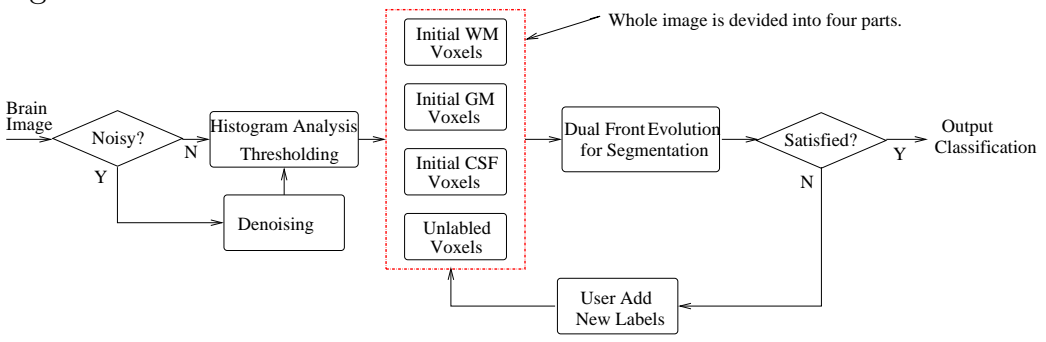

Fig. 1. Overall diagram of 3D brain cortex segmentation process. 


\subsection{Active Region and Potentials Decision By Histogram Analysis}

The original dual front active contours use morphological dilation to form the narrow active region from an initial curve. In fact, this is just one way to obtain the active region, we may use some other methods to define the active regions for a given segmentation, or class of images. And for the 3D brain tissue segmentation, the morphological dilatation is not a good solution because of the complex and convoluted structure of cortex. It is possible that the labels of some tiny and convoluted parts may be changed during dilation, and this will affect the result's accuracy.

In this subsection, we give a simple way to decide the active region by analyzing the histogram of the MRI normal brain image. The Fig. 2 shows the histogram analysis of the three tissues in brain: CSF, GM, and WM. Panel (a) is the histogram of a sample 3D MRI elderly brain image. There are three peaks and two bottoms in the histogram. These three peaks approximate to the average mean value of the three tissues. In fact, the neighborhoods of these two bottoms correspond to the voxels located around the boundary of different tissues. Because of the partial volume effect, it is hard to separate these voxels just by simple thresholding. We treat these voxels as unlabeled voxels, and use dual-front evolution to separate them.

As shown in the Panel (b) of Fig. 2, by setting different thresholds, the whole 3D image may be divided into GM seeds, WM seeds, CSF seeds, background points, and unlabeled voxels which are the voxels within the two regions $R_{1}$ and $R_{2}$ around these two bottoms. Background points are ignored for the computation. For the image with high noise level, we will smooth it first, then calculate the histogram and divide it.

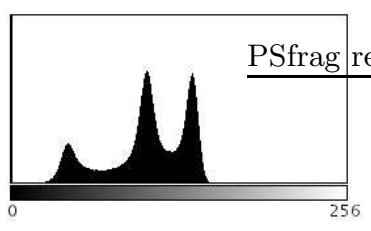

(a)

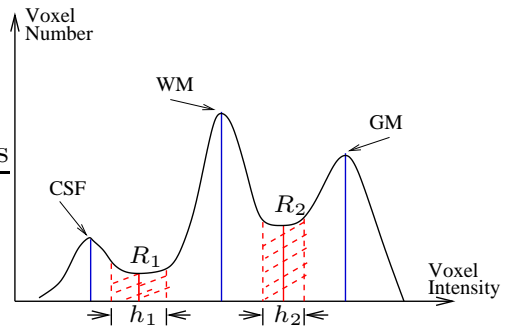

(b)

Fig. 2. The active region location is decided by the histogram analysis and thresholding of the 3D MRI brain image. (a): the histogram of a sample 3D MRI brain image; (b): the center of $R_{1}$ is the bottom between the peaks of CSF and WM, the center of $R_{2}$ is the bottom between the peaks of CSF and WM. $h_{1}$ and $h_{2}$ decide the size of $R_{1}$ and $R_{2}$.

Now, for the potentials for different labeled curves, we use region-based information for guiding the fronts' evolution. We calculate the mean values $u_{C S F}$, $u_{G M}$ and $u_{W M}$, the variance values $\sigma_{C S F}^{2}, \sigma_{G M}^{2}$ and $\sigma_{W M}^{2}$, of the three different kind of voxels with different labels $l_{C S F}, l_{G M}$ and $l_{W M}$. Then the propagation potentials for the labeled points $(x, y)$ are decided by

$$
\widetilde{P}_{l}(x, y)=w_{1} \cdot \exp \left(-\frac{\left|\bar{I}(x, y)-u_{l}\right|^{2}}{2 \sigma_{l}^{2}}\right)+w_{2} \quad \text { if } L(x, y)=l\left(l_{C S F}, l_{G M}, l_{W M}\right)
$$


where $\bar{I}(x, y)$ is the average value of the image intensity in a window of size $3 \times 3$ centered at the examined point.

\section{$3 \quad$ Experimental Results}

In this section, we show validations of our approach on various $3 \mathrm{D}$ simulated and real MRI brain image data sets. We use T1-weighted images for our test because they provide the best gray/white contrast and are commonly used for neuroanatomical analysis. All the experimental results shown in this section are obtained from 3D volume process directly.

To evaluate the efficiency of our method for every tissue type $T$ (GM, WM, and CSF), four probability measures are defined by equation

$T P=\frac{N_{B} \cap N_{R}}{N_{R}}, F N=\frac{N_{R}-N_{B} \cap N_{R}}{N_{R}}, F P=\frac{N_{B}-N_{B} \cap N_{R}}{N_{R}}, O M=\frac{T P}{1+F P}$,

where $N_{R}$ is the number of reference ground truth voxels of tissue $T . N_{B}$ is the number of voxels detected by our algorithm as the voxels of tissue $T$.

\subsection{Validation on Simulated MR brain data}

In Fig. 3, we present the results of the segmented WM tissues for five different slices of one 3D simulated brain image provided by BrainWeb [17]. We use the potentials defined by Eq. 1 with $\omega_{1}=1$ and $\omega_{2}=0.1$. The size of $R_{1}, h_{1}$, is equal to 20 , the size of $R_{2}, h_{2}$, is equal to 10 (see the Fig. 2).

In Table 1, we also give the comparison of the GM segmentation results on the same 3D simulated image of our scheme and other three methods, the fuzzy C-means method [18] implemented by ourselves, the Hidden Markov Method [12] provided by the website of FMRIB Software Library (http://www .fmrib.ox.ac . $\mathrm{uk} / \mathrm{fsl} /$ ), and the coupled surface algorithm reported in [7].

\begin{tabular}{|c|c|c|c|c|}
\hline Rate & $\begin{array}{c}\text { Fuzzy C-means } \\
\text { Method }\end{array}$ & $\begin{array}{c}\text { Hidden Markov } \\
\text { Method }\end{array}$ & $\begin{array}{c}\text { Coupled Surface } \\
\text { Algorithm }\end{array}$ & $\begin{array}{c}\text { Dual-Front } \\
\text { Active Contours }\end{array}$ \\
\hline TP (\%) & 87.3 & 92.3 & 92.8 & 93.3 \\
\hline FN (\%) & 12.7 & 7.7 & 7.2 & 6.7 \\
\hline FP (\%) & 23.2 & 5.7 & 6.0 & 5.6 \\
\hline Overlap Metric & 0.708 & 0.873 & 0.875 & 0.883 \\
\hline Time (s) & 373 & 500 & 3600 & 15 \\
\hline
\end{tabular}

Table 1. Comparison of the gray matter segmentation results of our method with some other methods on one 3D simulated brain image, which is the same one as that in Fig. 3.

In Fig. 4, the 3D models of segmented GM and WM surfaces from our method, and the ground truth data are also shown. From these segmentation results and comparison results, we can see that our scheme performs better than the other three methods in the accuracy of result and the computational efficiency.

We also test our method on 15 3D simulated brain images provided by BrainWeb [17,19], which are $T 1$ modality, $1 \mathrm{~mm}$ slice thickness, different noise levels 

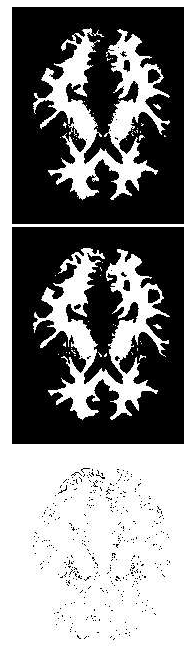

(a)
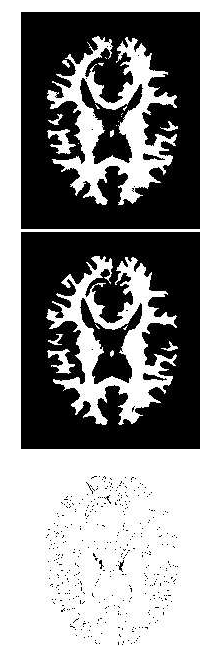

(b)
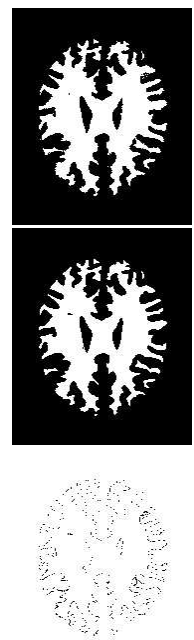

(c)
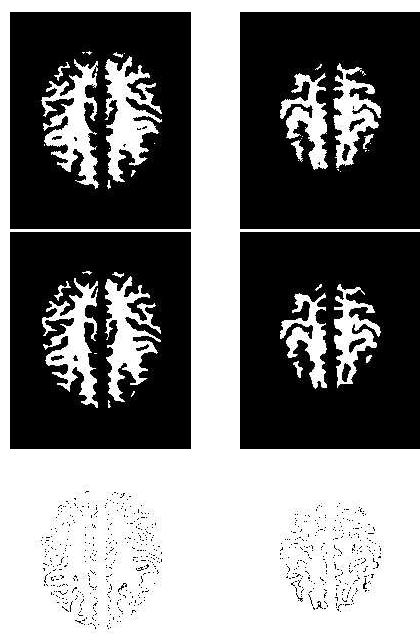

(d)

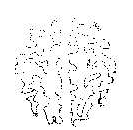

(e)

Fig. 3. Comparison of the segmentation results from our method with the ground truth data of five slices of one 3D simulated brain image provided by BrainWeb [17], which is $\mathrm{T} 1$ modality, $1 \mathrm{~mm}$ slice thickness, $3 \%$ noise level, $20 \%$ INU. And the image size is $181 \times 217 \times 181$.

The top row presents the segmentation results obtained from our method; the middle row shows the ground truth data provided by BrainWeb database; the bottom row shows the difference between the segmentation results from our method and the ground truth data. These five columns correspond to five slices of the test $3 \mathrm{D}$ image.

$1 \%, 3 \%, 5 \%, 7 \%$, and $9 \%$, and different INU settings $0 \%, 20 \%$, and $40 \%$. All images are the same size of $181 \times 217 \times 181$. We still use the potentials defined by Eq. 1 with $\omega_{1}=1$ and $\omega_{2}=0.1$. The size of $R_{1}, h_{1}$, is 20, the size of $R_{2}, h_{2}$ is 10 . For the image with high noise levels $5 \%, 7 \%$, and $9 \%$, we first use the isotropic nonlinear diffusion proposed by Perona and Malik [20] for denoising images. For the segmentation results, the overlap metrics of three tissues for these 15 images are from 0.747 to 0.944 . The measurement results show that our scheme performs well in segmenting the cortex even for the image with high noise level and INU setting.

\subsection{Validation on Real MR brain data}

To further evaluate our segmentation method under a wide range of imaging conditions, we also test the proposed algorithm on 20 real MRI brain images and compare the segmentation results with the manual segmentation by experts and some other segmentation methods. The 20 normal MR brain data sets and their manual segmentations were provided by the Center for Morphometric Analysis at Massachusetts General Hospital and are available at ISBR website http://www . cma.mgh.harvard.edu/ibsr/.

Fig. 5 shows the overlap metric, the average overlap metric of the CSF, GM and WM segmentation results on 20 normal brains, from the manual method, various automatic segmentation results provided by ISBR, the hidden Markov method [12] provided by the website of FMRIB Software Library (http://www . 


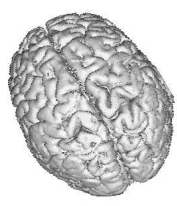

(a)

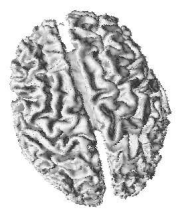

(b)

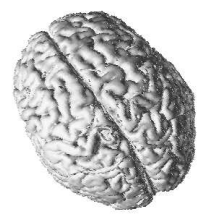

(c)

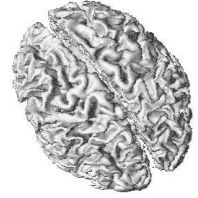

(d)

Fig. 4. Comparison of the 3D models of the GM and WM surfaces from our method and from the ground truth data. The test image is the same one as that in Fig. 3. (a) and (b) are the 3D models of the GM and WM surfaces obtained from our method; (c) and (d) are the 3D models of the GM and WM surfaces from the ground truth.

fmrib.ox.ac.uk/fsl/), and our proposed scheme. For the segmentation of these real brain images, we still use the potentials defined by Eq. 1 with $\omega_{1}=1$ and $\omega_{2}=0.1$. The size of $R_{1}, h_{1}$, is 20 , the size of $R_{2}, h_{2}$, is 10 . For these 20 brain images, the histograms are not as that shown in Fig. 2, we need to choose the two bottoms manually. So how to decide the two bottoms automatically need further research.

In this comparison with other segmentation methods, except for the methods provided by ISBR [21], we also compare our method with the other three recently proposed methods, the Bayesian method proposed in [11] (MPM-MAP); the couple-surface algorithm [7] (ZENG), and the hidden Markov method [12] (FAST). But with the rapid development of the medical image processing technology, it is impossible to compare all the related methods and test our methods on all the real brain images. This study is just the initial step of our research work on brain image analysis. We will still work on it, and improve the model's robustness and the segmentation's accuracy.

\subsection{Simple and Useful User Interaction}

The above two subsections show the segmentation result of our scheme with automatic thresholding only. Furthermore, our scheme is very convenient for users to add simple seed points just by mouse clicks to improve the segmentation accuracy dramatically. Fig. 6 gives an example of this nice property.

In Fig. 6, the first row shows one slice of the test 3D image (Panel (a)) which is the same as that in Fig. 3, the ground truth data of the WM tissue (Panel (b)) in this slice, and the corresponding 3D model of the WM tissue (Panel (c)). The second row shows the segmentation result based on one automatic thresholding. When setting the active region according to the Fig. 2, if the region $R_{2}$ is shifted to high intensity region, and the size of $R_{2}$ is large, we get the different active region between WM and GM tissue. The black voxels in Panel (d) present the unlabeled voxels after setting this threshold, and the different labeled voxels (shown in the figures with different colors) present different tissues' seed points. After segmentation, the WM tissue is shown in Panel (e), the 3D model of segmented WM is shown in Panel $(\mathrm{g})$. Here we can see, if automatic thresholding cannot provide enough WM seed points, the segmented WM tissue is incorrect. In this case, the third row shows the segmentation result after user interaction. As shown in Panel (h), the user interaction is just several mouse clicks within seconds to add some new seed points manually only on one slice, then we run the dual-front 


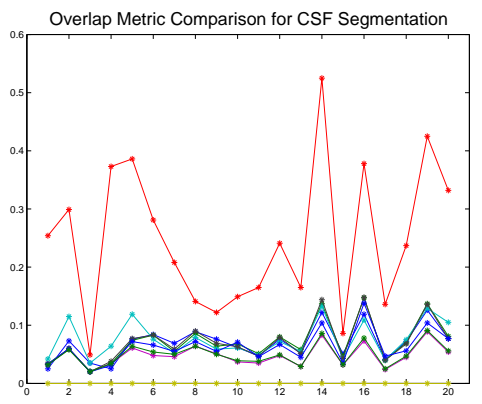

(a)

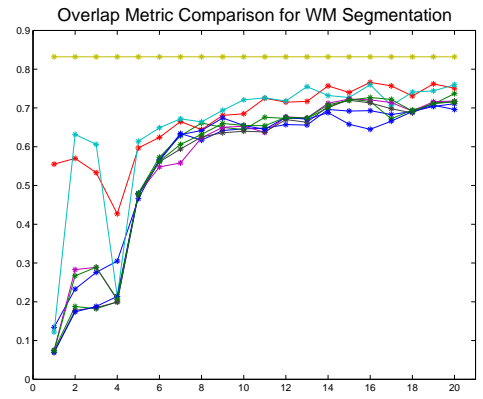

(d)

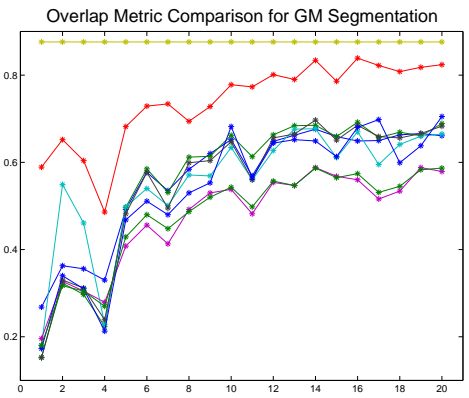

(b)

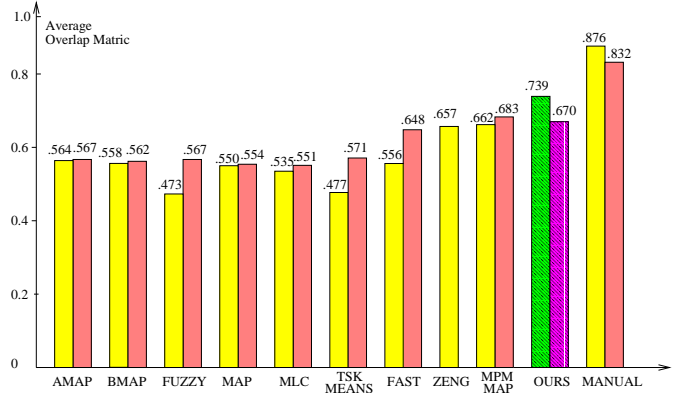

(e)

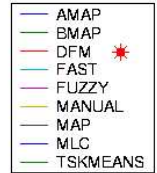

(c)

Fig. 5. The overlap metric of the CSF, GM, and WM segmentation results on 20 normal real brain images from various segmentation methods. The results of some automatic segmentation methods provided by ISBR. AMAP: adaptive MAP; BMAP: biased MAP; FUZZY: fuzzy c-means; MAP: Maximum Aposteriori Probability; MLC: MaximumLikelihood; TSKMEANS: tree-structure k-means; FAST: Hidden Markov method [12]; MPM-MAP: the Bayesian method proposed in [11]; ZENG: the couple-surface algorithm [7]; DFM: our scheme.

evolution again to segment GM and WM. The segmented boundary of GM/WM is shown in Panel (i), the extracted WM tissue and the corresponding 3D model are shown in Panel (j) and Panel (k). The figures show that the accuracy of the result after user interaction is much better than that just based on automatic thresholding. This sample shows that our method allow the simple user-interaction, we will still research on the efficiency of the user-interaction.

\subsection{Computational Time}

Another nice property of our methods is the high computational efficiency. We test our method on 15 simulated 3D MR brain images and 20 real normal 3D MR brain images. The average total computation time is around 20 seconds, in which the average computational time for the histogram analysis is around 5 seconds and the average computational time for dual front evolution is around 15 seconds, on the $2.5 \mathrm{GHz}$ Pentium4 PC processor.

We downloaded the software of the Hidden Markovian method from the website of FMRIB Software Library (http://www.fmrib.ox.ac.uk/fsl/), and implemented the fuzzy C-means method [18] for the comparison with our method. On the same computer, the average computational time of Hidden Markovian method for the same test images used in our method is around 550 seconds, the 


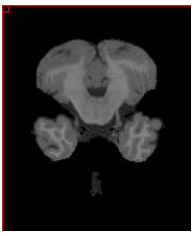

(a)

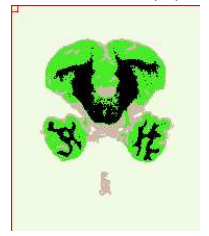

(d)

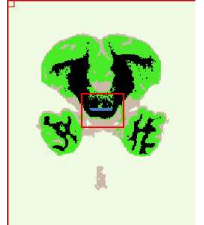

(h)

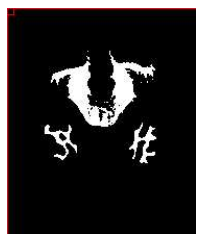

(b)

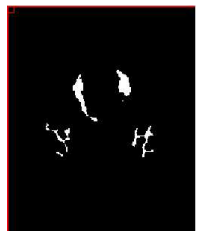

(f)

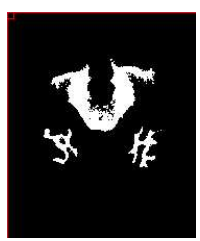

(j)

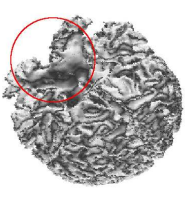

$(\mathrm{k})$

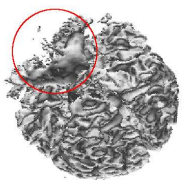

(c)

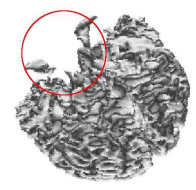

(g)

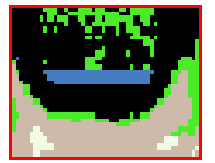

(l)

Fig. 6. The simple user interaction can improve the segmentation accuracy dramatically. The blue line in figure (l) is the zoom-in of the user added seed points in figure $(\mathrm{h})$. The difference of the segmentation result from the automatic thresholding (fig.(g)), the automatic thresholding with manual correction (fig.(k)), and the ground truth (fig.(c)) are shown in the red circles of figure $(\mathrm{c}),(\mathrm{g})$, and $(\mathrm{k})$.

average computational time of the fuzzy C-means method for the same images is around 450 seconds. The comparison shows that our method is much faster then these two methods. The average overlap metric for gray matter and white matter segmentation of these two methods are 0.473 and 0.567 from fuzzy C-means methods, 0.556 and 0.648 from Hidden Markov method, which are lower than that of 0.739 and 0.670 from our method.

Although the average overlap metric for white matter segmentation of the Bayesian method proposed in [11] on the same 20 real brain data sets is 0.683 (shown in Fig. 5), a little higher than that of 0.670 from our method, they reported that the computational time is around 280 seconds which is much slower than our methods.

Because many active contour model based methods always combined with some pre-processing methods such as fuzzy C-means, Bayesian segmentation, and etc. The active contour model is just implemented as the final step to yield the final cortical surface, it is hard for us to give an exact comparison. Here, we give the brief discussion about the computational time of some active contour model based methods.

Xu's method [6] combined the adaptive fuzzy C-means algorithm [9], they reported that the computational time for the final deformable surface algorithm is about 3 hours. As for the coupled surface method proposed by Zeng [7], it was reported that for a $3 \mathrm{D}$ image of the whole brain with the voxel size of $1.2 \times 1.2 \times$ 
$1.2 \mathrm{~mm}^{3}$, their algorithm runs in about $1 \mathrm{~h}$ on a SGI Inigo2 machine with a $195 \mathrm{MHz}$ R10000 processor for the implementation of skull stripping, cortex segmentation and measurement simultaneously. They also reported that the average overlap metric for gray matter segmentation on the same 20 normal brain images provided by ISBR is 0.657 , which is lower than that of our method, 0.739. Goldenberg [8] also adopted the coupled surfaces principle and used the fast geodesic active contour approach to improve the computational time for cortex segmentation. They reported that the computational time of their method is about 2.5 mins for a $192 \times 250 \times 170$ MR image of the whole brain on a Pentium3 PC. But they did not give the quantitative analysis of the segmentation results.

\section{Conclusions and Future Work}

In this paper, we propose a novel scheme for 3D brain cortex segmentation based on dual-front active contours. The cortex segmentation results from the synthetic and real MR brain images, and the computation time compared with other methods demonstrate the high reconstruction accuracy, the low computational cost and the minimum user interaction of this scheme. In the future, our research work will focus on the following aspects.

First, our dual-front active contour model is very fast, and easy to be implemented, it is flexible to combine with other methods as the post-processing method and improve the segmentation accuracy further. We will work on the combination of the current model with some powerful INU bias compensation methods and smooth methods to improve the model's robustness.

Second, in this paper, we just use the region-based information to guide the curve evolution because the interfaces among difference tissues is not clear (because of the partial volume effect). We are also working on proposing some better local operators, and combine them with the region-based information in the potentials to improve the accuracy of the segmentation result further.

Third, our model can be generalized to multi-spectral data, which is very common in MR imaging. In this case, the voxel intensities are assume to be vectors instead of scalars, and how to design the potentials, and the thresholds need further investigation.

Fourth, we are working on finding the better methods to choose active region automatically for improving the method's generality.

This dual front active contour model provides a novel, simple idea for 3D brain tissue segmentation, and also has strong potential applications in other medical image analysis domains, where a volumetric layer is the study of interest. Examples include the left ventricular (LV) myocardium of the heart and the boundary of the liver.

\section{References}

1. Kass, M., Witkin, A., Terzopoulos, D.: Snakes: Active contour models. International Journal of Computer Vision 1 (1988) 321-332 
2. Malladi, R., Sethian, J.: A geometric approach to segmentation and analysis of $3 \mathrm{~d}$ medical images. In: Processings of Mathematical Methods in Biomedical Image Analysis, MMBIA'96. (1996)

3. Davatzikos, C., Bryan, R.: Using a deformable surface model to obtain a shape representation of the cortex. IEEE Trans. on Medical Imaging 15 (1996) 785-795

4. Teo, P., Sapiro, G., Wandell, B.: Creating connected representations of cortical gray matter for functional mri visualization. IEEE Trans. on Medical Imaging 16 (1997) 852-863

5. Dale, A., Fischl, B., Sereno, M.: Cortical surface-based analysis. NeuroImage 9 (1999) 179-194

6. Xu, C., Pham, D., Rettmann, M., Yu, D., Prince, J.: Reconstruction of the human cerebral cortex from magnetic resonance images. IEEE Trans. on Medical Imaging 18 (1999) 467-480

7. Zeng, X., Staib, L., Schultz, R., Duncan, J.: Segmentation and measurement of the cortex from 3D MR images using coupled surfaces propagation. IEEE Transactions on Medical Imaging 18 (1999) 100-111

8. Goldenberg, R., Kimmel, R., Rivlin, E., Rudzsky, M.: Cortex segmentation: A fast variational geometric approach. IEEE Trans. on Medical Imaging 21 (2002) 15441551

9. Pham, D., Prince, J.: Adaptive fuzzy segmentation of magnetic resonance images. IEEE Trans. on Medical Imaging 18 (1999) 737-752

10. Liew, A., Yan, H.: An adaptive spatial fuzzy clustering algorithm for $3 \mathrm{~d} \mathrm{mr}$ image segmentation. IEEE Trans. on Medical Imaging 22 (2003) 1063-1075

11. Marroquin, J., Vemuri, B., et al.: An accurate and efficient bayesian method for automatic segmentation of brain mri. IEE Trans. on Medical Imaging 21 (2002) 934-945

12. Zhang, Y., Brady, M., Smith, S.: Segmentation of brain mr images through a hidden markov random field model and the expectation maximization algorithm. IEEE Trans. on Medical Imaging 20 (2001) 45-57

13. Ruan, S., Moretti, B., Fadili, J., Bloyet, D.: Fuzzy markovian segmentation in application of magnetic resonance images. Computer Vision and Image Understanding 85 (2002) 54-69

14. Leemput, K.V., Maes, F., Vandermeulen, D., Suetens, P.: Automated model-based tissue classification of $\mathrm{mr}$ images of the brain. IEEE Trans. on Medical Imaging $\mathbf{1 8}$ (1999) 897-908

15. Kapur, T., Grimson, W., et al.: Segmentation of brain tissue from magnetic resonance images. Medical Image Analysis 1 (1996) 109-127

16. Li, H., Elmoataz, A., Fadili, J., Ruan, S.: Dual front evolution model and its application in medical imaging. In: MICCAI2004. Volume 3216., Rennes/Saint-Malo, France (2004) 103-110

17. BrainWeb: Mcconnell brain imaging center, montreal neurological institute. (http://www.bic.mni.mcgill.ca/brainweb/)

18. Bezdek, J.: Pattern Recognition with Fuzzy Objective Functions Algorithms. Plenum (1981)

19. Cocosco, C., Kollokian, V., Kwan, R., Evans, A.: Brainweb: Online interface to a 3D MRI simulated brain database. NeuroImage 5 (1997) S425

20. Perona, P., Malik, J.: Scale-space and edge detection using anisotropic diffusion. IEEE Trans. on PAMI 12 (1990) 629-639

21. Filipek, P., Richelme, C., Kennedy, D., Caviness, V.: The young adult human brain: an mri-based morphometric analysis. Cerebral Cortex 4 (1994) 344-360 